\title{
Article \\ Nutritional Status of Children with Cerebral Palsy in Gorkha, Nepal: Findings from the Nepal Cerebral Palsy Register
}

\author{
Israt Jahan 1,2,3,*, Mohammad Muhit 1,2, Mahmudul Hassan Al Imam 1,2,3,4, Ratul Ghose 1,2, \\ Amir Banjara Chhetri ${ }^{5}$, Nadia Badawi ${ }^{6,7}$ and Gulam Khandaker ${ }^{3,4,8}$ \\ 1 CSF Global, Dhaka 1213, Bangladesh; mmuhit@hotmail.com (M.M.); physiomahmud@yahoo.com (M.H.A.I.); \\ splash348@gmail.com (R.G.) \\ 2 Asian Institute of Disability and Development (AIDD), University of South Asia, Dhaka 1212, Bangladesh \\ 3 School of Health, Medical and Applied Sciences, Central Queensland University, \\ Rockhampton, QLD 4701, Australia; gulam.khandaker@health.nsw.gov.au \\ 4 Central Queensland Public Health Unit, Central Queensland Hospital and Health Service, \\ Rockhampton, QLD 4700, Australia \\ 5 CSF Global-Nepal, Balaju, Kathmandu 44611, Nepal; amirbanjara@yahoo.com \\ 6 Cerebral Palsy Alliance Research Institute, The University of Sydney, Sydney, NSW 2086, Australia; \\ nadia.badawi@health.nsw.gov.au \\ 7 Grace Centre for Newborn Intensive Care, Sydney Children's Hospital Network, \\ Westmead, NSW 2145, Australia \\ 8 Discipline of Child and Adolescent Health, Sydney Medical School, The University of Sydney, \\ Sydney, NSW 2006, Australia \\ check for \\ * Correspondence: arda.jahan89@gmail.com; Tel.: +880-173-900-2305
}

updates

Citation: Jahan, I.; Muhit, M.; Al Imam, M.H.; Ghose, R.; Chhetri, A.B.;

Badawi, N.; Khandaker, G.

Nutritional Status of Children with

Cerebral Palsy in Gorkha, Nepal:

Findings from the Nepal Cerebral Palsy Register. Nutrients 2021, 13, 2537. https://doi.org/10.3390/ nu13082537

Academic Editors: Patrick R. M. Lauwers and Josep A. Tur

Received: 21 June 2021

Accepted: 19 July 2021

Published: 25 July 2021

Publisher's Note: MDPI stays neutral with regard to jurisdictional claims in published maps and institutional affiliations.

Copyright: (C) 2021 by the authors Licensee MDPI, Basel, Switzerland. This article is an open access article distributed under the terms and conditions of the Creative Commons Attribution (CC BY) license (https:// creativecommons.org/licenses/by/ $4.0 /)$.

\begin{abstract}
Background: The study aimed to define the burden and underlying risk factors of malnutrition among children with cerebral palsy (CP) in Gorkha district, Nepal. Methods: The first population-based register of children with CP in Gorkha, Nepal (i.e., Nepal CP Register-NCPR) was established in 2018. Children aged $<18$ years with confirmed CP were registered following standard protocol. Nutritional status was determined based on anthropometric measurements (height/length, weight, mid-upper-arm-circumference) following WHO guidelines. Descriptive analyses and adjusted logistic regression were completed. Results: Between June-October 2018, 182 children with CP were registered into the NCPR (mean (SD) age at assessment: 10.3 (5.0) years, 37.4\% female). Overall, $51.7 \%, 64.1 \%$, and $29.3 \%$ children were underweight, stunted, and thin, respectively. Furthermore, $14.3 \%$ of children with CP aged $<5$ years had severe wasting. Underweight and stunting were significantly higher among children with spastic CP $(p=0.02, p<0.001)$ and/or Gross Motor Function Classification System (GMFCS) level (III-V) $(p=0.01, p<0.001)$ and/or who were not enrolled in school ( $p=0.01, p<0.001)$. In adjusted analysis, GMFCS level III-V and non-attendance to school significantly increased the odds of stunting by 8.2 (95\% CI 1.6, 40.8) and 4.0 (95\% CI 1.2, 13.2) times, respectively. Conclusions: the high rate of different forms of undernutrition among children with $\mathrm{CP}$ in Gorkha, Nepal is concerning. Need-based intervention should be taken as priority to improve their nutritional outcome.
\end{abstract}

Keywords: cerebral palsy (CP); malnutrition; disability; key informant method; Nepal

\section{Introduction}

Cerebral Palsy $(\mathrm{CP})$ is a non-progressive neurological condition that results from lesion to the developing brain [1]. The impact of $\mathrm{CP}$ on a child's life is multidimensional and not limited to functional impairment. The motor impairment also affects nutritional status, quality of life, oral health and even survival probability of a child with CP [2-6]. Maintenance of adequate nutrition is critical and malnutrition is common among this vulnerable population [2-4,7-9]. Although in recent years increased number of studies 
have focused on the nutritional status of children with CP [2-4,8-12], the evidence gap in low- and middle-income countries (LMICs) is still high.

In a recent population-based study in Bangladesh, the prevalence of $\mathrm{CP}$ was estimated at 3.4 per 1000 live births which is nearly twice that estimated globally [13,14]. In the same cohort, $70 \%$ of children were underweight and/or stunted [2]. A similar high burden of malnutrition was also reported from Indonesia and Uganda [3,9]. The causal pathway to malnutrition among children with $\mathrm{CP}$ is yet not clearly defined particularly in LMIC settings, though several studies found a significant association between malnutrition, severity of motor impairment (e.g., Gross Motor Function Classification System (GMFCS) level III-V, tri/quadriplegia) and associated impairments (e.g., intellectual, speech, hearing) among children with CP $[2-4,7,15]$

In Nepal, very little is known about the epidemiology of CP. The limited available institution-based studies indicate a substantial higher burden of low economic status, delayed diagnosis, and severe motor impairment among children with $\mathrm{CP}$ in the country [16-18]. However, none of the available studies reported the nutritional status of children with CP in Nepal. This gap in evidence on the epidemiology of malnutrition (e.g., burden, preventable and manageable risk factors) remains a major obstacle for planning targeted nutritional intervention for children with $\mathrm{CP}$ in a geographically complex LMIC such as Nepal. Population-based studies hold great potential to contribute reliable evidence and serve as baseline data for such intervention studies as well as program planning. This study aimed to describe the burden and underlying risk factors of malnutrition among children with CP using population-based data in remote Gorkha district of Nepal.

\section{Materials and Methods}

\subsection{Participants and Settings}

This study used data from the first population-based register of children with $\mathrm{CP}$ ( $<18$ years) in six municipalities of remote Gorkha district, Nepal, i.e., Nepal CP Register-NCPR. The NCPR was established in 2018 and the study area encompasses $\sim 823 \mathrm{sq} \mathrm{km}$ area and 46,681 households with a total population $\sim 184,546$ (child population aged $<18$ years $~ 83,047)[19,20]$

The community-based key informant method (KIM, a validated and widely used method) $[3,13,21]$ was used to identify children with suspected CP. A total of 174 key informants (KIs-local volunteers) were trained as part of NCPR. Following training, the KIs received six weeks to identify children with suspected $\mathrm{CP}$ and share their name and contact information with the community mobilizers (CM, i.e., paid project staff). The CMs then, with help from the KIs, brought all these children and their primary caregivers to medical camps. Each child underwent detailed neurodevelopmental assessment by a multidisciplinary medical team including a pediatrician, a nutritionist, and a physiotherapist for confirmed clinical diagnosis, registration into the NCPR and data collection [13]. The clinical definition of CP used in NCPR was adopted from the Bangladesh Cerebral Palsy Register (BCPR), [13] based on the Surveillance of Cerebral Palsy in Europe (SCPE) [22] and the Australian Cerebral Palsy Register (ACPR) [23].

\subsection{Measures}

Detailed information about socio-demographic characteristics, pre- and perinatal history, motor function severity, associated impairments, anthropometric measurements, and educational status of the children registered into the NCPR (i.e., children with confirmed $\mathrm{CP}$ ) were collected. Structured guidelines and a data collection template adopted from BCPR and ACPR were used [13,23].

\subsection{Clinical Assessment}

GMFCS and Manual Ability Classification System (MACS) were used to assess and define motor function severity following standard guidelines [24,25]. The Viking Speech Scale (VSS) and Communication Function Classification System (CFCS) were used to describe 
the motor speech disorder and functional communication of registered children [26,27]. Presence and severity of associated impairments were determined based on clinical examination, review of available medical records and detailed history provided by the primary caregivers. All assessments were completed following the BCPR protocol [13].

\subsection{Anthropometric Measurements}

Weight: Weight was measured in kilograms using a digital weighing scale with a precision of $10 \mathrm{~g}$ following the WHO protocol [28]; three repeated measures were taken, and the average was documented. Tared weight was measured for young children aged $<2$ years and children who had difficulties in standing independently.

Length/height: Recumbent length/height was measured in centimeters (cm). Recumbent length was measured for children aged $<2$ years using a manual length board with a precision of $1 \mathrm{~cm}$. For children aged $\geq 2$ years, height was measured using a height board following WHO protocol [28]. Segmental measurement i.e., knee height, was measured for children who could not stand independently due to muscle stiffness, weakness or deformities. The full body length/height was estimated using following formula; height $=(2.69 \times$ knee height $)+24.2$ in $\mathrm{cm} \mathrm{[29]}$.

Mid-upper arm circumference (MUAC): MUAC was measured in $\mathrm{cm}$ using MUAC tape following the WHO protocol [28].

\subsection{Indicators Used to Define the Nutritional Status of Children}

The following indicators were used to measure the nutritional status of children registered in the NCPR, (i) weight-for-age z score (WAZ), (ii) height-for-age z score (HAZ), (iii) weight-for-height $\mathrm{z}$ score (WHZ), (iv) BMI-for-age z score (BAZ), and (v) MUAC-for-age $\mathrm{z}$ score (MUACZ). All z scores were calculated using WHO Anthro and WHO AnthroPlus software. HAZ and BAZ were calculated for children aged $<18$ years, WAZ was calculated for children aged $<10.1$ years, and WHZ and MUACZ were calculated for children aged $<5.1$ years. The $\mathrm{z}$ scores were categorized using the WHO cut-off values (overnutrition: $\mathrm{z}$ score $>+2$ SD, normal: $\mathrm{z}$ score -2.0 to $+2.0 \mathrm{SD}$, moderate undernutrition: $\mathrm{z}$ score $\geq-3.0$ to $<-2.0 \mathrm{SD}$, severe undernutrition: $\mathrm{z}$ score $<-3.0 \mathrm{SD}$ ) to determine the nutritional status of children [28]. A child was considered underweight if WAZ $<-2.0 \mathrm{SD}$, stunted if $\mathrm{HAZ}<-2.0 \mathrm{SD}$, wasted if $\mathrm{WHZ}$ or MUACZ $<-2.0 \mathrm{SD}$, and thin if $\mathrm{BAZ}<-2.0 \mathrm{SD}$. Venn diagram was used to show the overlapping of different forms of undernutrition among the participating children.

\subsection{Statistical Analysis}

Data were analyzed using SPSS (IBM Corporation, Chicago, IL, USA) version 26. Skewness and Kurtosis were used to examine the distribution of continues variables, e.g., age, monthly family income, z scores. Descriptive analyses were completed using valid percentages to report the overall nutritional status and bivariate analyses were used to identify potential determinants of undernutrition among children in NCPR. Chi-squared test and Fisher's exact test were used. Factors that were found significantly related to underweight and/or stunting and/or thinness among participating children in the cross tabulation (e.g., chi-square, fisher's exact test) were included in unadjusted logistic model as potential predictors. Factors significantly associated with underweight or stunted or thinness in unadjusted analyses were entered into the adjusted model i.e., logistic regression; adjusted odds ratios (aOR) with $95 \%$ confidence intervals (95\% CIs) were reported. A $p<0.05$ was considered significant.

\section{Results}

Between June and October 2018, 213 children with suspected CP underwent detailed neurodevelopmental assessment in 13 medical assessment camps. Of these, 182 had a clinically confirmed diagnosis of CP. The mean (SD) age at assessment was 10.3 (5.0) years, $37.4 \%(n=68)$ female. Of all, $45.1 \%(n=82 / 182)$ were living in the rural areas. 


\subsection{Overall Nutritional Status}

Over two thirds $(78.2 \%, n=136 / 174$, missing data, $n=8)$ of the children had at least one form of malnutrition. Of all, $51.7 \%(n=45 / 87)$ were underweight (i.e., WAZ $<-2 \mathrm{SD})$, $64.1 \%(n=109 / 170)$ were stunted (i.e., HAZ $<-2 \mathrm{SD})$ and $29.3 \%(n=48 / 164)$ were thin (i.e., BAZ $<-2 \mathrm{SD})$. Among children aged $\leq 5$ years, $14.3 \%(n=4 / 28)$ had severe wasting i.e., severe acute malnutrition (SAM) according to MUACZ (i.e., MUACZ $<-3 S$ ); however, this proportion was $7.7 \%(n=2 / 26)$ when WHZ was used as an indicator (i.e., $\mathrm{WHZ}<-3 \mathrm{SD}$ ). The mean (SD) and median [inter quartile range (IQR)] of the $\mathrm{z}$ scores have been summarized in Table 1 .

Table 1. Overall nutritional status of children registered into the Nepal CP Register (NCPR).

\begin{tabular}{|c|c|c|c|c|c|}
\hline Indicator & $\begin{array}{l}\text { Weight-for-Age z } \\
\text { Score (WAZ) }\end{array}$ & $\begin{array}{l}\text { Height-for-Age z } \\
\text { Score (HAZ) }\end{array}$ & $\begin{array}{l}\text { BMI-for-Age z } \\
\text { Score (BAZ) }\end{array}$ & $\begin{array}{l}\text { Weight-for- } \\
\text { Height-z Score } \\
\text { (WHZ) }\end{array}$ & $\begin{array}{l}\text { MUAC-for-Age-z } \\
\text { Score (MUACZ) }\end{array}$ \\
\hline$n$ & $87^{1}$ & $170^{2}$ & $164^{3}$ & $26^{2,4}$ & $28^{2,4}$ \\
\hline Mean (SD) & $-2.2(1.9)$ & $-2.9(2.6)$ & $-0.5(4.1)$ & $-0.5(1.6)$ & $-0.9(1.4)$ \\
\hline Median (IQR) & $-2.1(-3.8,-0.9)$ & $-2.8(-4.5,-1.4)$ & $-1.1(-2.5,0.6)$ & $-0.1(-1.7,0.5)$ & $-0.6(-2.2,0.1)$ \\
\hline $\begin{array}{l}\text { Overnutrition, } n(\%) \\
\text { (z score: }>+2 \text { SD) }\end{array}$ & $4(4.6)$ & $6(3.5)$ & $13(7.9)$ & $1(3.8)$ & $0(0.0)$ \\
\hline $\begin{array}{c}\text { Normal, } n(\%) \\
\text { (z score: }-2 \text { SD to }+2 \text { SD) }\end{array}$ & $38(43.7)$ & $55(32.4)$ & $103(62.8)$ & $21(80.8)$ & $21(75.0)$ \\
\hline $\begin{array}{l}\text { Moderate undernutrition, } n(\%) \\
\text { (z score: }>-3 \text { SD to }<-2.0 \text { SD) }\end{array}$ & $11(12.6)$ & 30 (17.6) & 19 (11.6) & $2(7.7)$ & 3 (10.7) \\
\hline $\begin{array}{l}\text { Severe undernutrition, } n(\%) \\
\quad(\mathrm{z} \text { score: } \leq-3.0 \mathrm{SD})\end{array}$ & $34(39.1)$ & $79(46.5)$ & $29(17.7)$ & $2(7.7)$ & $4(14.3)$ \\
\hline
\end{tabular}

${ }^{1}$ Weight for age was calculated for children aged 0-121 months; ${ }^{2}$ Missing data; ${ }^{3}$ Weight for height was calculated for children aged 0-61 months; ${ }^{4}$ MUAC for age z score was calculated for children aged 0-61 months.

\subsection{Socio-Demographic Characteristics and Nutritional Status of Children with $C P$}

Table 2 summarizes the relationship between socio-demographic characteristics and nutritional status of children with $\mathrm{CP}$ in the study area. No significant difference in underweight and stunting was observed among children with $\mathrm{CP}$ with changes in educational level of father $(p=0.58$ and $p=0.87$, respectively), and mother $(p=0.06$ and $p=0.05$, respectively), occupation of father ( $p=0.38$ and $p=0.48$, respectively) and mother ( $p=0.70$ and $p=0.29$, respectively), source of drinking water ( $p=0.72$ and $p=0.76$, respectively) and access to sanitation ( $p=0.60$ and $p=0.33$, respectively). Similar observations were made for thinness among children. However, underweight was more prevalent among children with low monthly family income compared to others in the cohort $(59.1 \%$ if $<150$ USD vs. $13.6 \%$ if $\geq 300$ USD; $p=0.03$ ). A similar pattern was observed for stunting and thinness, but this relationship was not statistically significant ( $p=0.09$ and $p=0.93$ respectively). Furthermore, school enrolment was significantly lower among school-aged children who were underweight $(p=0.01)$, stunted $(p<0.001)$ and thin $(p=0.02)$ in the NCPR cohort.

\subsection{Clinical Characteristics and Nutritional Status of Children with $C P$}

\subsubsection{Birthweight and Gestational Age}

All children with CP in NCPR who had low birth weight also showed stunting $(p<0.001)$. However, nutritional status of children did not vary with their gestational age. (Table 3) 
Table 2. Weight-for-age, height-for-age and BMI-for-age of children registered into NCPR according to their socio-demographic characteristics.

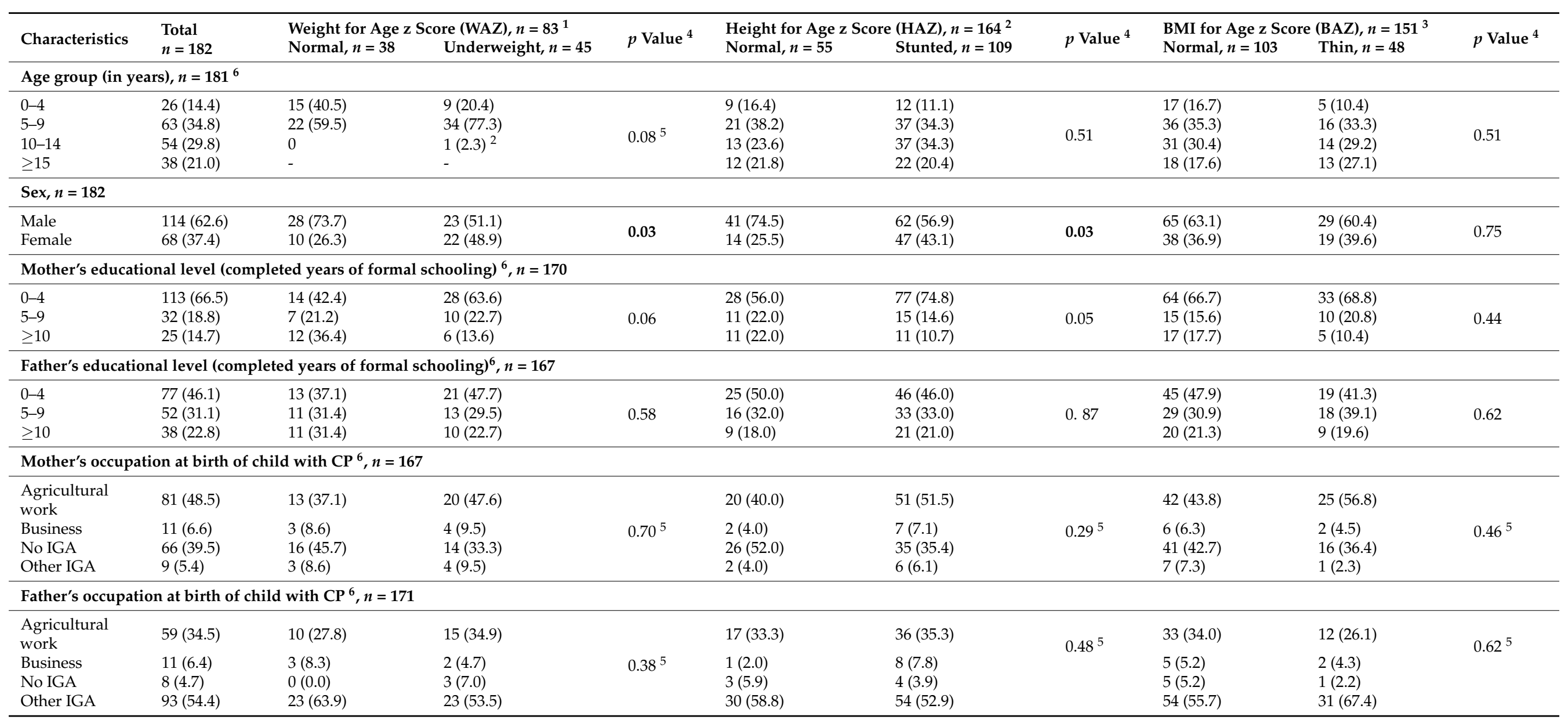


Table 2. Cont.

\begin{tabular}{|c|c|c|c|c|c|c|c|c|c|c|}
\hline \multirow{2}{*}{ Characteristics } & \multirow{2}{*}{$\begin{array}{l}\text { Total } \\
n=182\end{array}$} & \multicolumn{2}{|c|}{ Weight for Age z Score (WAZ), $n=83^{1}$} & \multirow{2}{*}{$p$ Value $^{4}$} & \multicolumn{2}{|c|}{ Height for Age z Score (HAZ), $n=164^{2}$} & \multirow{2}{*}{$p$ Value $^{4}$} & \multicolumn{2}{|c|}{ BMI for Age z Score (BAZ), $n=151^{3}$} & \multirow{2}{*}{$p$ Value $^{4}$} \\
\hline & & Normal, $n=38$ & Underweight, $n=45$ & & Normal, $n=55$ & Stunted, $n=109$ & & Normal, $n=103$ & Thin, $n=48$ & \\
\hline \multicolumn{11}{|c|}{ Monthly family income, NRs (USD) ${ }^{6,7}, n=181$} \\
\hline Median (IQR) & $\begin{array}{l}10,000(7000 \\
20,000)(82 \\
(57,164))\end{array}$ & $\begin{array}{l}17,000(9750,20,500) \\
(140(80.0168))\end{array}$ & $\begin{array}{l}12,000(7000,20,000) \\
(98(57,164))\end{array}$ & 0.14 & $\begin{array}{l}12,000(8000,20,000) \\
(98(66,164))\end{array}$ & $\begin{array}{l}10,500(7000,20,000) \\
(86(57,164))\end{array}$ & 0.88 & $\begin{array}{l}12,000(7000,20,000) \\
(98(57,164)\end{array}$ & $\begin{array}{l}13,500(8250,20,000) \\
(111(68,164))\end{array}$ & 0.55 \\
\hline $\begin{array}{l}1000-14,999 \\
(10-149.9)\end{array}$ & $103(56.9)$ & $13(34.2)$ & $26(59.1)$ & \multirow[b]{2}{*}{$0.03^{5}$} & $29(52.7)$ & $62(57.4)$ & \multirow[b]{2}{*}{0.09} & $54(52.9)$ & $24(50.0)$ & \multirow[b]{2}{*}{0.93} \\
\hline $\begin{array}{l}15,000-29,999 \\
(150-299.9)\end{array}$ & $61(33.7)$ & $21(55.3)$ & $12(27.3)$ & & $24(43.6)$ & $33(30.6)$ & & $37(36.3)$ & 19 (39.6) & \\
\hline \multicolumn{11}{|c|}{ Source of drinking water ${ }^{6}, n=177$} \\
\hline $\begin{array}{l}\text { Improved }^{8} \\
\text { Unimproved }\end{array}$ & $\begin{array}{l}163(92.6) \\
13(7.4) \\
\end{array}$ & $\begin{array}{l}31(86.1) \\
5(13.9) \\
\end{array}$ & $\begin{array}{l}40(90.9) \\
4(9.1) \\
\end{array}$ & $0.72^{5}$ & $\begin{array}{l}48(90.6) \\
5(9.4) \\
\end{array}$ & $\begin{array}{l}99(92.5) \\
8(7.5) \\
\end{array}$ & $0.76^{5}$ & $\begin{array}{l}91(91.9) \\
8(8.1) \\
\end{array}$ & $\begin{array}{l}43(89.6) \\
5(10.4) \\
\end{array}$ & $0.76^{5}$ \\
\hline \multicolumn{11}{|c|}{ Type of latrin used ${ }^{6}$} \\
\hline $\begin{array}{l}\text { Sanitary }{ }^{9} \\
\text { Non-sanitary }\end{array}$ & $\begin{array}{l}169(97.1) \\
5(2.9) \\
\end{array}$ & $\begin{array}{l}34(94.4) \\
2(5.6) \\
\end{array}$ & $\begin{array}{l}40(97.6) \\
1(2.4) \\
\end{array}$ & $0.60^{5}$ & $\begin{array}{l}48(94.2) \\
3(5.8)\end{array}$ & $\begin{array}{l}103(98.1) \\
2(1.9)\end{array}$ & $0.33^{5}$ & $\begin{array}{l}95(96.9) \\
3(3.1) \\
\end{array}$ & $\begin{array}{l}44(95.7) \\
2(4.3)\end{array}$ & $0.65^{5}$ \\
\hline \multicolumn{11}{|c|}{ Attendance at mainstream and/or special school ${ }^{6}, n=174$} \\
\hline
\end{tabular}

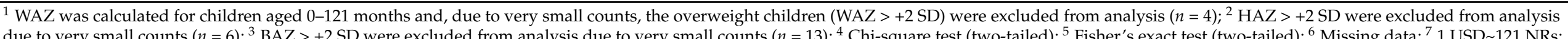

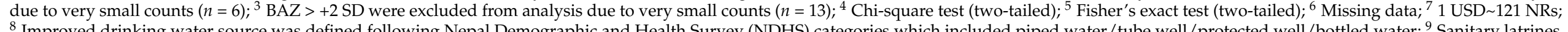
included flush toilet/pit latrine. The $p$ values in 'bold' font indicate significant difference. 
Table 3. Weight-for-age, height-for-age and BMI-for-age of children registered into the NCPR according to their clinical characteristics.

\begin{tabular}{|c|c|c|c|c|c|c|c|c|c|c|}
\hline $\begin{array}{l}\text { Risk Factors and } \\
\text { Clinical Characteristics }\end{array}$ & $\begin{array}{l}\text { Total, } \\
n=182\end{array}$ & $\begin{array}{l}\text { Weight for Age } \\
\text { Normal, } n=38\end{array}$ & $\begin{array}{c}\text { ore }\left(\text { WAZ) }{ }^{1}, n=83\right. \\
\text { Underweight, } n=45\end{array}$ & $p$ Value $^{4}$ & $\begin{array}{l}\text { Height for Age z } \\
\text { Normal, } n=55\end{array}$ & $\begin{array}{c}\text { ore }\left(\text { HAZ) }{ }^{2}, n=164\right. \\
\text { Stunted, } n=109\end{array}$ & $p$ Value $^{4}$ & $\begin{array}{l}\text { BMI for Age z Sc } \\
\text { Normal, } n=103\end{array}$ & $\begin{array}{l}(\mathrm{BAZ})^{3}, n=151 \\
\text { Thin, } n=48\end{array}$ & $p$ Value $^{4}$ \\
\hline \multicolumn{11}{|c|}{ Birthweight (BW) among known ${ }^{6}, n=78$} \\
\hline $\begin{array}{l}\text { Normal BW } \\
\text { Low BW }\end{array}$ & $\begin{array}{l}57(73.1) \\
21(26.9)\end{array}$ & $\begin{array}{l}19(79.2) \\
5(20.8)\end{array}$ & $\begin{array}{l}19(57.6) \\
14(42.4)\end{array}$ & 0.09 & $\begin{array}{l}22(100.0) \\
0(0.0)\end{array}$ & $\begin{array}{l}29(60.4) \\
19(39.6)\end{array}$ & 0.001 & $\begin{array}{l}35(74.5) \\
12(25.5)\end{array}$ & $\begin{array}{l}14(66.7) \\
7(33.3)\end{array}$ & 0.51 \\
\hline \multicolumn{11}{|c|}{ Gestational age ${ }^{6}, n=170$} \\
\hline $\begin{array}{l}\text { Pre-term } \\
\text { Term }\end{array}$ & $\begin{array}{l}18(10.6) \\
152(89.4)\end{array}$ & $\begin{array}{l}4(12.1) \\
29(87.9)\end{array}$ & $\begin{array}{l}8(18.6) \\
35(81.4)\end{array}$ & 0.44 & $\begin{array}{l}3(6.1) \\
46(93.9)\end{array}$ & $\begin{array}{l}15(14.6) \\
88(85.4)\end{array}$ & 0.13 & $\begin{array}{l}14(14.7) \\
81(85.3)\end{array}$ & $\begin{array}{l}3(6.5) \\
43(93.5)\end{array}$ & 0.16 \\
\hline $\begin{array}{l}\text { No } \\
\text { Yes }\end{array}$ & $\begin{array}{l}132(72.5) \\
50(27.5)\end{array}$ & $\begin{array}{l}24(63.2) \\
14(36.8)\end{array}$ & $\begin{array}{l}33(73.3) \\
12(26.7)\end{array}$ & 0.32 & $\begin{array}{l}41(74.5) \\
14(25.5)\end{array}$ & $\begin{array}{l}75(68.8) \\
34(31.2)\end{array}$ & 0.45 & $\begin{array}{l}78(75.7) \\
25(24.3)\end{array}$ & $\begin{array}{l}31(64.6) \\
17(35.4)\end{array}$ & 0.15 \\
\hline \multicolumn{11}{|l|}{ Intellectual ${ }^{6}, n=132$} \\
\hline $\begin{array}{l}\text { No } \\
\text { Yes }\end{array}$ & $\begin{array}{l}47(35.6) \\
85(64.4)\end{array}$ & $\begin{array}{l}14(50.0) \\
14(50.0)\end{array}$ & $\begin{array}{l}9(26.5) \\
25(73.5)\end{array}$ & 0.06 & $\begin{array}{l}21(51.2) \\
20(48.8)\end{array}$ & $\begin{array}{l}23(28.7) \\
57(71.3)\end{array}$ & 0.01 & $\begin{array}{l}35(46.1) \\
41(53.9)\end{array}$ & $\begin{array}{l}9(27.3) \\
24(72.7)\end{array}$ & 0.07 \\
\hline \multicolumn{11}{|l|}{ Visual $^{6}, n=179$} \\
\hline $\begin{array}{l}\text { No } \\
\text { Yes }\end{array}$ & $\begin{array}{l}161(89.9) \\
18(10.1)\end{array}$ & $\begin{array}{l}32(84.2) \\
6(15.8)\end{array}$ & $\begin{array}{l}37(84.1) \\
7(15.9)\end{array}$ & 1.00 & $\begin{array}{l}49(92.5) \\
4(7.5)\end{array}$ & $\begin{array}{l}95(88.0) \\
13(12.0)\end{array}$ & 0.38 & $\begin{array}{l}91(90.1) \\
10(9.9)\end{array}$ & $\begin{array}{l}41(87.2) \\
6(12.8)\end{array}$ & 0.60 \\
\hline $\begin{array}{l}\text { No } \\
\text { Yes }\end{array}$ & $\begin{array}{l}143(79.9) \\
36(20.1)\end{array}$ & $\begin{array}{l}29(76.3) \\
9(23.7)\end{array}$ & $\begin{array}{l}33(73.3) \\
12(26.7)\end{array}$ & 0.76 & $\begin{array}{l}42(79.2) \\
11(20.8)\end{array}$ & $\begin{array}{l}86(78.9) \\
23(21.1)\end{array}$ & 0.96 & $\begin{array}{l}83(81.4) \\
19(18.6)\end{array}$ & $\begin{array}{l}36(78.3) \\
10(21.7)\end{array}$ & 0.66 \\
\hline \multicolumn{11}{|l|}{ Speech $^{6}, n=181$} \\
\hline $\begin{array}{l}\text { No } \\
\text { Yes }\end{array}$ & $\begin{array}{l}36(19.9) \\
145(80.1)\end{array}$ & $\begin{array}{l}10(26.3) \\
28(73.7)\end{array}$ & $\begin{array}{l}6(13.3) \\
39(86.7)\end{array}$ & 0.13 & $\begin{array}{l}16(29.6) \\
38(70.4)\end{array}$ & $\begin{array}{l}18(16.5) \\
91(83.5)\end{array}$ & 0.05 & $\begin{array}{l}26(25.5) \\
76(74.5)\end{array}$ & $\begin{array}{l}6(12.5) \\
42(87.5)\end{array}$ & 0.07 \\
\hline \multicolumn{11}{|c|}{ Number of associated impairments, $n=182$} \\
\hline $\begin{array}{l}\text { None } \\
\leq 2 \\
\text { Multiple }\end{array}$ & $\begin{array}{l}23(12.6) \\
113(62.1) \\
46(25.3) \\
\end{array}$ & $\begin{array}{l}6(15.8) \\
20(52.6) \\
12(31.6) \\
\end{array}$ & $\begin{array}{l}5(11.1) \\
26(57.8) \\
14(31.1) \\
\end{array}$ & 0.80 & $\begin{array}{l}12(21.8) \\
33(60.0) \\
10(18.2) \\
\end{array}$ & $\begin{array}{l}9(8.3) \\
68(62.4) \\
32(29.4) \\
\end{array}$ & 0.03 & $\begin{array}{l}17(16.5) \\
65(63.1) \\
21(20.4) \\
\end{array}$ & $\begin{array}{l}5(10.4) \\
28(58.3) \\
15(31.3) \\
\end{array}$ & 0.28 \\
\hline \multicolumn{11}{|l|}{ Timing of $\mathrm{CP}^{6}, n=180$} \\
\hline $\begin{array}{l}\text { Pre \& Peri } \\
\text { Postnatal }\end{array}$ & $\begin{array}{l}159(88.3) \\
21(11.7)\end{array}$ & $\begin{array}{l}31 \text { (81.6) } \\
7(18.4)\end{array}$ & $\begin{array}{l}42(93.3) \\
3(6.7)\end{array}$ & $0.17^{5}$ & $\begin{array}{l}45(83.3) \\
9(16.7)\end{array}$ & $\begin{array}{l}99(91.7) \\
9(8.3)\end{array}$ & 0.12 & $\begin{array}{l}88(88.1) \\
12(11.9)\end{array}$ & $\begin{array}{l}43 \text { (89.6) } \\
5(10.4)\end{array}$ & 0.78 \\
\hline
\end{tabular}


Table 3. Cont.

\begin{tabular}{|c|c|c|c|c|c|c|c|c|c|c|}
\hline \multirow{2}{*}{$\begin{array}{l}\text { Risk Factors and } \\
\text { Clinical Characteristics }\end{array}$} & \multirow{2}{*}{$\begin{array}{l}\text { Total, } \\
n=182\end{array}$} & \multicolumn{2}{|c|}{ Weight for Age z Score (WAZ) ${ }^{1}, n=83$} & \multirow{2}{*}{$p$ Value $^{4}$} & \multicolumn{2}{|c|}{ Height for Age z Score (HAZ) ${ }^{2}, n=164$} & \multirow{2}{*}{$p$ Value $^{4}$} & \multicolumn{2}{|c|}{ BMI for Age z Score $(\mathrm{BAZ})^{3}, n=151$} & \multirow{2}{*}{$p$ Value $^{4}$} \\
\hline & & Normal, $n=38$ & Underweight, $n=45$ & & Normal, $n=55$ & Stunted, $n=109$ & & Normal, $n=103$ & Thin, $n=48$ & \\
\hline \multicolumn{11}{|c|}{ Swallowing difficulties ${ }^{6}, n=179$} \\
\hline No & $117(65.4)$ & $24(63.2)$ & $24(54.5)$ & 0.43 & $40(74.1)$ & $67(61.5)$ & 0.11 & $69(67.6)$ & $29(61.7)$ & 0.48 \\
\hline \multicolumn{11}{|c|}{ Predominant type of $\mathrm{CP}, n=182$} \\
\hline Spastic & $141(77.5)$ & $24(63.2)$ & $39(86.7)$ & \multirow{3}{*}{$0.02^{5}$} & $39(70.9)$ & 89 (81.7) & \multirow{3}{*}{$<0.001^{5}$} & $74(71.8)$ & $40(83.3)$ & \multirow{3}{*}{$0.42^{5}$} \\
\hline Dyskinesia & $7(3.8)$ & $2(5.3)$ & $0(0.0)$ & & $2(3.6)$ & $4(3.7)$ & & $4(3.9)$ & $2(4.2)$ & \\
\hline Ataxia & $16(8.8)$ & 7 (18.4) & $1(2.2)$ & & $12(21.8)$ & $2(1.8)$ & & $11(10.7)$ & $3(6.3)$ & \\
\hline \multicolumn{11}{|c|}{ Topography of CP, $n=141$} \\
\hline Mono/Hemiplegia & $54(38.3)$ & $10(41.7)$ & $11(28.2)$ & \multirow{3}{*}{$0.54^{5}$} & $24(61.5)$ & $26(29.2)$ & \multirow{3}{*}{$0.002^{5}$} & $31(41.9)$ & $12(30.0)$ & \multirow{3}{*}{$0.18^{5}$} \\
\hline Diplegia & $16(11.3)$ & $3(12.5)$ & $5(12.8)$ & & $4(10.3)$ & 10 (11.2) & & 10 (13.5) & $3(7.5)$ & \\
\hline Tri/Quadriplegia & $71(50.4)$ & $11(45.8)$ & $23(59.0)$ & & $11(28.2)$ & $53(59.6)$ & & $33(44.6)$ & $25(62.5)$ & \\
\hline \multicolumn{11}{|c|}{$\mathrm{CP}$ diagnosis age (in months) ${ }^{6}, n=168$} \\
\hline$\leq 30$ & $78(46.4)$ & $19(52.8)$ & $18(46.2)$ & \multirow{3}{*}{0.85} & $21(42.9)$ & $48(46.2)$ & \multirow{3}{*}{0.02} & $39(41.5)$ & $23(50.0)$ & \multirow{3}{*}{0.54} \\
\hline $31-60$ & $38(22.6)$ & $8(22.2)$ & 10 (25.6) & & $6(12.2)$ & $30(28.8)$ & & $23(24.5)$ & $8(17.4)$ & \\
\hline$\geq 61$ & $52(31.0)$ & $9(25.0)$ & $11(28.2)$ & & $22(44.9)$ & $26(25.0)$ & & $32(34.0)$ & $15(32.6)$ & \\
\hline I-II & $82(45.1)$ & $22(57.9)$ & $13(28.9)$ & \multirow[b]{2}{*}{0.01} & 37 (67.3) & 37 (33.9) & \multirow[b]{2}{*}{$<0.001$} & 54 (52.4) & 15 (31.3) & \multirow[b]{2}{*}{0.01} \\
\hline III-V & $100(54.9)$ & $16(42.1)$ & $32(71.1)$ & & $18(32.7)$ & $72(66.1)$ & & $49(47.6)$ & $33(68.8)$ & \\
\hline MACS level, $n=177$ & & & & & & & & & & \\
\hline I-II & $88(49.7)$ & $17(47.2)$ & $16(37.2)$ & & $35(66.0)$ & 45 (42.5) & & 58 (58.6) & $17(35.4)$ & \\
\hline III-V & $72(40.7)$ & $10(27.8)$ & $21(48.8)$ & 0.14 & $12(22.6)$ & 55 (51.9) & 0.002 & $32(32.3)$ & $26(54.2)$ & 0.02 \\
\hline Not applicable ${ }^{7}$ & $17(9.6)$ & $9(25.0)$ & $6(14.0)$ & & $6(11.3)$ & $6(5.7)$ & & $9(9.1)$ & $5(10.4)$ & \\
\hline Viking speech level $^{6}, r$ & $=180$ & & & & & & & & & \\
\hline I-II & $61(33.9)$ & 12 (31.6) & $11(24.4)$ & & $25(46.3)$ & $32(29.6)$ & & 40 (39.6) & $14(29.2)$ & \\
\hline III-IV & $102(56.7)$ & 17 (44.7) & $28(62.2)$ & 0.25 & $23(42.6)$ & 70 (64.8) & 0.02 & $52(51.5)$ & $29(60.4)$ & 0.46 \\
\hline Not applicable & $17(9.4)$ & $9(23.7)$ & $6(13.3)$ & & $6(11.1)$ & $6(5.6)$ & & $9(8.9)$ & $5(10.4)$ & \\
\hline CFCS level $^{6}, n=180$ & & & & & & & & & & \\
\hline I-II & $82(45.6)$ & $20(52.6)$ & $15(33.3)$ & 0.08 & $32(59.3)$ & 43 (39.8) & 0.02 & 55 (54.5) & 17 (35.4) & 0.03 \\
\hline III-V & $98(54.4)$ & 18 (47.4) & 30 (66.7) & & $22(40.7)$ & $65(60.2)$ & & 46 (45.5) & 31 (64.6) & \\
\hline
\end{tabular}

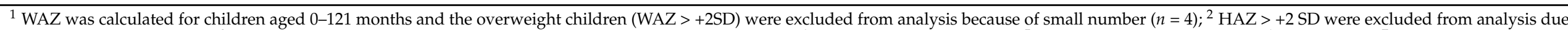

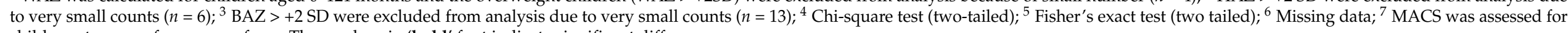
children at or over four years of age. The $p$ values in 'bold' font indicate significant difference. 


\subsubsection{Timing of $\mathrm{CP}$ and Age of $\mathrm{CP}$ Diagnosis}

There was no significant difference in WAZ, HAZ and BAZ according to the timing of $\mathrm{CP}(p=0.17, p=0.12, p=0.78$, respectively) of children. (Table 3$)$

Although underweight and thinness were observed slightly higher among children who were diagnosed with $\mathrm{CP}$ before 30 months of age than those diagnosed after 60 months of age, the differences were not statistically significant $(46.2 \%$ vs. $28.2 \%, p=0.85$ and $50.0 \%$ vs. $32.6 \%, p=0.54$, respectively). Stunting, on the other hand, were significantly overrepresented among children diagnosed with $\mathrm{CP}$ aged 0-30 months than others in the cohort $(p=0.02)$. (Table 3$)$

3.3.3. Predominant Type, Topography, Motor Function Severity, Motor Speech Disorder and Functional Communication

Underweight and stunting were more prevalent among children with spastic $\mathrm{CP}$ ( $p=0.02$ and $p<0.001$, respectively). Moreover, stunting was overrepresented among children with tri/quadriplegia and children with VSS level III-V ( $p=0.002$ and $p=0.02$ respectively). Furthermore, stunting and thinness were comparatively higher among children with MACS level III-V ( $p=0.002$ and $p=0.02$ respectively) and CFCS level III-V ( $p=0.02$ and $p=0.03$ respectively). All three forms of undernutrition (i.e., underweight, stunting, thinness) were significantly higher among children with GMFCS level III-V $(p=0.01, p<0.001, p=0.01$ respectively). (Table 3$)$

A significant positive relationship between the 'GMFCS level and nutritional status' and 'nutritional status and school attendance' was also observed among the participating children. Both school attendance and nutritional status was considerably low among children with GMFCS level III-V than children with GMFCS level I-II (Tables 2, 3 and S1).

\subsubsection{Associated Impairments}

Underweight, stunting and thinness were overrepresented among children with at least one associated impairment. Furthermore, stunting was more prevalent among children with intellectual impairment $(p=0.01)$. However, no significant relationship between nutritional status and other associated impairments (e.g., epilepsy, visual, hearing, speech impairment) were observed in the cohort ( $p \geq 0.05$ for all). (Table 3 )

\subsection{Predictors of Underweight, Stuntin and Thinness Among Children with CP \\ 3.4.1. Underweight}

In unadjusted analysis, sex, attendance to mainstream school and GMFCS level were found to be significantly associated with underweight. When fitted in an adjusted model, none of those factors remained significant $(p=0.30, p=0.08, p=0.16$, respectively). (Tables S2 and 4 )

Table 4. Predictors of underweight, stunting and thinness among children with CP in Gorkha, Nepal (Adjusted analysis).

\begin{tabular}{|c|c|c|c|c|c|c|}
\hline \multirow{2}{*}{ Predictors $^{1}$} & \multicolumn{2}{|c|}{ Underweight (WAZ $<-2 \mathrm{SD}$ ), $n=45$} & \multicolumn{2}{|c|}{ Stunting $(\mathrm{HAZ}<-2 \mathrm{SD}), n=109$} & \multicolumn{2}{|c|}{ Thin $(\mathrm{BAZ}<-2 \mathrm{SD}), n=48$} \\
\hline & aOR $[95 \% \mathrm{CI}]$ & $p$ Value & aOR $[95 \% \mathrm{CI}]$ & $p$ Value & aOR $[95 \% \mathrm{CI}]$ & $p$ Value \\
\hline \multicolumn{7}{|l|}{ Sex } \\
\hline Male & $\operatorname{Ref}$ & & $\operatorname{Ref}$ & & - & \\
\hline Female & $2.1(0.5,9.2)$ & 0.30 & $1.8(0.6,5.6)$ & 0.28 & - & \\
\hline \multicolumn{7}{|c|}{ Attendance to mainstream school } \\
\hline Yes & Ref & & Ref & & Ref & \\
\hline No & $3.8(0.9,16.8)$ & 0.08 & $4.0(1.2,13.2)$ & 0.02 & $2.2(0.7,6.3)$ & 0.15 \\
\hline \multicolumn{7}{|l|}{ GMFCS level } \\
\hline I-II & Ref & & Ref & & Ref & \\
\hline III-V & $2.9(0.6,13.0)$ & 0.16 & $8.2(1.6,40.8)$ & 0.01 & $1.6(0.6,4.1)$ & 0.31 \\
\hline
\end{tabular}


Table 4. Cont.

\begin{tabular}{|c|c|c|c|c|c|c|}
\hline \multirow{2}{*}{ Predictors ${ }^{1}$} & \multicolumn{2}{|c|}{ Underweight (WAZ $<-2$ SD), $n=45$} & \multicolumn{2}{|c|}{ Stunting (HAZ $<-2 \mathrm{SD}), n=109$} & \multicolumn{2}{|c|}{ Thin (BAZ $<-2$ SD), $n=48$} \\
\hline & aOR $[95 \% \mathrm{CI}]$ & $p$ Value & aOR $[95 \% \mathrm{CI}]$ & $p$ Value & aOR $[95 \% \mathrm{CI}]$ & $p$ Value \\
\hline \multicolumn{7}{|l|}{ MACS level } \\
\hline $\begin{array}{l}\text { I-II } \\
\text { III-V }\end{array}$ & - & & $\begin{array}{l}\text { Ref } \\
0.1(0.0,0.9)\end{array}$ & 0.04 & - & \\
\hline \multicolumn{7}{|l|}{ CFCS level } \\
\hline $\begin{array}{l}\text { I-II } \\
\text { III-V }\end{array}$ & - & & $\begin{array}{l}\text { Ref } \\
1.7(0.4,7.1)\end{array}$ & 0.44 & $\begin{array}{l}\text { Ref } \\
1.3(0.5,3.2)\end{array}$ & 0.58 \\
\hline \multicolumn{7}{|c|}{ Viking speech level } \\
\hline $\begin{array}{l}\text { I-II } \\
\text { III-V }\end{array}$ & - & & $\begin{array}{l}\operatorname{Ref} \\
1.2(0.3,4.3)\end{array}$ & 0.77 & - & \\
\hline \multicolumn{7}{|c|}{ Intellectual impairment } \\
\hline $\begin{array}{l}\text { No } \\
\text { Yes }\end{array}$ & - & & $\begin{array}{l}\operatorname{Ref} \\
2.5(0.8,8.2)\end{array}$ & 0.11 & - & \\
\hline
\end{tabular}

\subsubsection{Stunting}

In case of stunting, sex, attendance to mainstream school, GMFCS level, MACS level, VSS level, CFCS level and intellectual impairment were identified as significant predictors. When adjusted for other covariates, attendance to mainstream school, GMFCS level and MACS level of child were found to be significantly associated with stunting. Children who never attended mainstream school and children with GMFCS level III-V had 4.0 $(95 \%$ CI 1.2, 13.2) and $8.2(95 \%$ CI 1.6, 40.8) times higher odds of stunting when compared to others in the cohort, respectively. (Tables S2 and 4)

\subsubsection{Thinness}

In unadjusted analysis, the odds of being thin was significantly higher among children with GMFCS level III-V, MACS level III-V, CFCS level III-V, and those who were not enrolled in mainstream school. When fitted in adjusted model, none of those predictors remained significant. (Tables S2 and 4)

\subsection{Children with Multiple Forms of Undernutrition}

In the NCPR cohort, 12 children had underweight, stunting and thinness. The majority had the most severe forms of motor impairment (e.g., GMFCS level III-V: $n=10 / 12$; tri/quadriplegia: $n=9 / 12$ ), severe speech motor disorder (VSS level III-V: $n=10 / 12$ ) and low functional communication level (CFCS level III-V: $n=9 / 12$ ), associated impairments (e.g., multiple associated impairments: $n=4 / 12$ ). Furthermore, all were from ultra-poor families and half $(n=6 / 12)$ had never received any rehabilitation services. (Figure 1 ) 


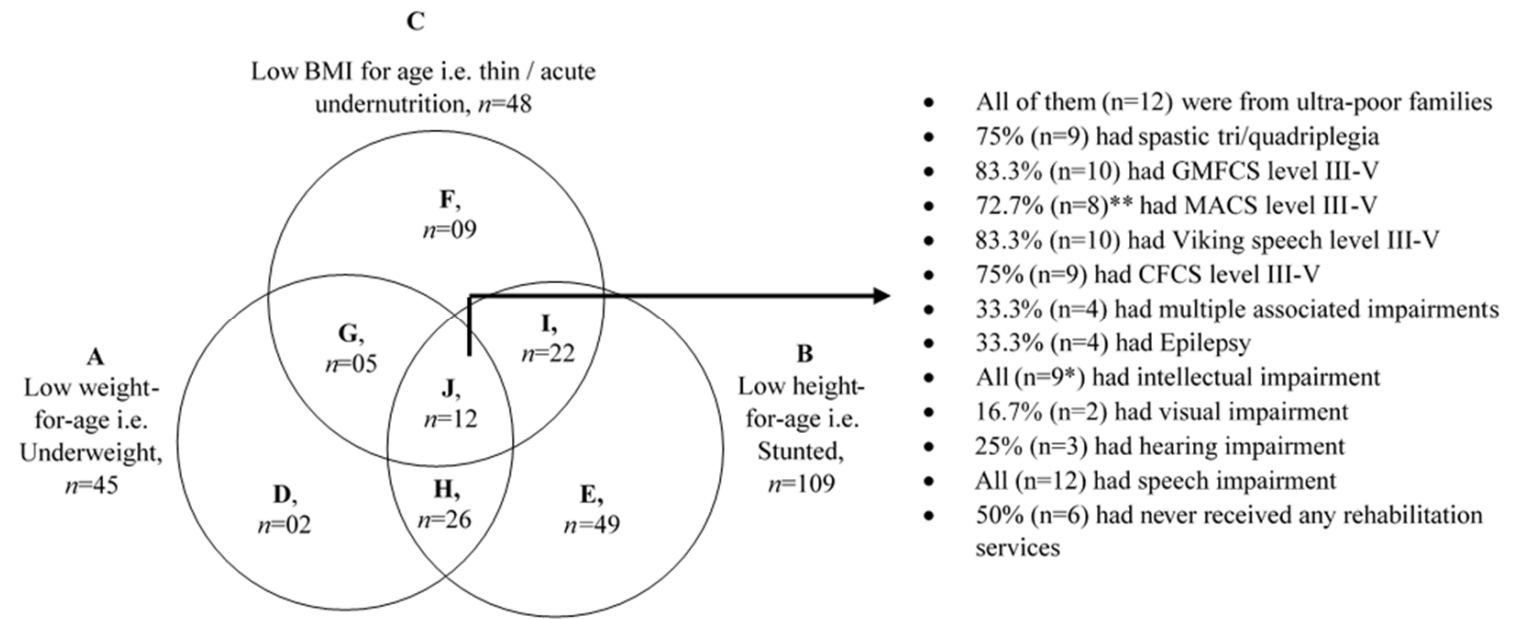

Figure 1. Overlapping of different forms of undernutrition among children with $\mathrm{CP}$ in NCPR. Here, (A) = Total number of children who were underweight with or without other forms of undernutrition; $(B)=$ total number of children who were stunted with or without other forms of undernutrition; $(C)=$ total number of children who were thin with or without other forms of undernutrition; (D) = children who were underweight only; $(\mathrm{E})=$ children who were stunted only; $(\mathrm{F})=$ children who were thin only; $(\mathrm{G})=$ children who were both underweight and thin; $(\mathrm{H})=$ children who were both underweight and stunted; (I) = children who were both stunted and thin; $(\mathrm{J})$ children who had all three forms of undernutrition (i.e., underweight, stunting, thinness); ${ }^{*}$ Missing data; ${ }^{* *}$ was assessed for children aged at or over 4 years.

\section{Discussion}

To our knowledge, this is the first population-based study reporting nutritional status of children with $\mathrm{CP}$ in Nepal. The study revealed a high burden of malnutrition among children with CP in remote Gorkha, Nepal. Nearly two-thirds of children in NCPR had at least one form of malnutrition, and more than one-third had severe undernutrition. When compared to regional data, a considerably higher rate of wasting was observed among children with $\mathrm{CP}$ aged $<5$ years than the general population of similar age [30]. A similar high burden was reported in other LMICs $[2-4,9,31]$.

Malnutrition among children with $\mathrm{CP}$ is complex and is due to multiple interlinked factors. It is evident that children with severe motor impairments are the most vulnerable. Several studies have reported a strong relationship between the GMFCS level and malnutrition among children with CP in both LMICs and HICs [2,4,15,32]. Similar findings have been observed in this study. There is a two way relationship between motor severity and malnutrition among children with CP. Children with severe CP (e.g., GMFCS level III-V) are likely to have severe feeding difficulties, poor digestive capacity and altered nutritional requirement [31-37]. These factors directly affect the nutrient intake, and thus their nutritional status. On the other hand, malnutrition impairs the cerebral function of children, weakens their immune system to fight against infections, and interferes in nutritional intake/absorption causing growth failure [37]. Early identification of malnutrition and comprehensive nutritional support is necessary to break this perpetual relationship and avert the adverse consequences of malnutrition among children with CP. Considering the complex geography of the study site, it is likely that most children in NCPR did not have access to health care and rehabilitation services as needed.

Moreover, children who had speech impairment and severe communication limitations had poor nutritional status than others in the cohort. Communication plays a vital role in maintaining daily activities including feeding management of children with $\mathrm{CP}$. Although not significant, the nutritional status of children with intellectual disabilities was comparatively poor than others in NCPR. Advancement in assistive technologies and evidence-based guidelines developed in recent decades have enhanced opportunities for learning and active participation of children with $\mathrm{CP}$ in daily activities, thus improving quality of life. However, such facilities are rarely accessible to children from low socio- 
economic status and remote settings like Gorkha. The findings suggest that children who were attending schools had a slightly better nutritional status than those not attending. This could be explained by low school attendance and higher malnutrition rates observed among children with severe GMFCS level in the NCPR cohort.

A high proportion of stunting and underweight was observed among children who had LBW and/or were born preterm. Similar positive correlation was reported previously among children with CP in Bangladesh and Indonesia [2,3]. LBW is considered as one of the major predisposing factors for chronic undernutrition, i.e., stunting among children. The recent Nepal Demographic and Health Survey (NDHS) also indicates a high proportion of malnutrition among children who had very small size at birth [30].

Nutrition is crucial and influences every aspect of life to different extents. Because of the long-term impact on health, quality of life and survival, it is important to address malnutrition at an early stage of life and break the vicious cycle. With the growing evidence regarding vulnerability of children with $\mathrm{CP}$, it is now apparent that there is substantial need for action and implementation of evidence-based programming to improve their nutritional status. Up to now, several interventions have been proposed to improve the nutritional outcome of children with CP in HICs and LMICs [37-39]. However, in LMICs strategies mostly focused on behavioral interventions, e.g., training to the caregivers on feeding skills, nutrition education $[38,39]$, and the impact of such interventions on nutritional outcome of children with CP in LMICs like Nepal is unknown. Communitybased nutrition intervention/programs focusing on need and nutritional outcome could be beneficial.

In recent decades Nepal has showed remarkable strides in reducing the burden of chronic malnutrition among children nationally [30]. Combating childhood malnutrition is one of the key priorities of the government of Nepal, and several initiatives have been undertaken to improve the current scenario. Nevertheless, it is not clear where children with disability, e.g., CP, stand in this agenda. Although the government of Nepal has initiated a social security scheme for people with disability i.e., disability card and pension, several challenges in accessing these services have been reported [40]. The key challenge remains covering the evidence gap, coordination, and collaboration in translational research for children with disability in Nepal.

\section{Strengths and Limitations}

The main strength of this study is that it used population-based data to report the burden and the underlying factors of malnutrition among children with $\mathrm{CP}$ in the remote Gorkha district of Nepal. The use of multiple indicators assisted in data triangulation, identification of children suffering from acute and chronic malnutrition, and potential predictors of malnutrition, using adjusted analysis. However, despite considerable effort, this study has several limitations. First, weight, height and MUAC were used to assess nutritional status, which have limitations in the precise estimation of the nutritional status of children with CP; therefore, triceps skin-fold thickness and arm fat area are considered as recommended measurements to assess this nutritional status [37]. However, considering the resource constraints (i.e., trained professional, standard skinfold calipers) and complex study settings, these were the optimal choice to minimize error and ensure the quality of the collected data. Second, the anthropometric data were compared to WHO reference curves developed for the general population as a $\mathrm{CP}$ specific growth chart is not available for children in LMICs. Third, the causal pathway to malnutrition among children with $\mathrm{CP}$ in Gorkha, Nepal, could not be established as it was beyond the scope of this cross-sectional study design. Fourth, the study sites (i.e., Gorkha) represent the hilly regions of the country, therefore the findings reported in this study are not generalizable to the other parts of the country (e.g., plain land/Tarai). However, this study described the epidemiology of malnutrition among children with CP in Gorkha, Nepal, and these findings will help plan future research and nutrition interventions in such complex settings as that of Nepal. 


\section{Conclusions}

The burden of malnutrition is high among children with CP in Gorkha, Nepal. Although Nepal has demonstrated progress in addressing the challenges toward improving nutritional status of children, disability specific data are lacking. The study findings have identified potential predictors of malnutrition, essential for development of need-based nutrition intervention strategies for this vulnerable group of population. The findings are important for program planners and policy makers working in the disability sector in Nepal to prioritize inclusion of these vulnerable children, enhancing opportunity for their development and promotion of a disability inclusive society in Nepal.

Supplementary Materials: The following are available online at https://www.mdpi.com/article/ 10.3390/nu13082537/s1, Table S1: Relationship between GMFCS level, school attendance (among children aged $\geq 5$ years) and nutritional status of children with CP in NCPR, Table S2: Predictors of underweight, stunting and thinness among children with $\mathrm{CP}$ in Gorkha, Nepal (Unadjusted analysis).

Author Contributions: Conceptualization, G.K., M.M. and N.B.; methodology, G.K., M.M. and N.B.; software, I.J. and G.K.; validation, I.J. and G.K.; formal analysis, I.J. and G.K.; investigation, G.K., R.G., M.H.A.I.; resources, I.J., G.K. and M.M.; data curation, I.J., R.G., M.H.A.I., A.B.C. and G.K.; writing — original draft preparation, I.J.; writing—review and editing, All authors; visualization, I.J., G.K.; supervision, G.K. and M.M.; project administration, A.B.C.; funding acquisition, G.K. and N.B. All authors have read and agreed to the published version of the manuscript.

Funding: This research was funded by the Cerebral Palsy Alliance Research Foundation (grant number: PG10017) and internal funding from CSF Global in Bangladesh and Nepal.

Institutional Review Board Statement: The study was conducted in accordance with the "Helsinki Declaration of 1975 (as revised in 1983)". Ethical approval was obtained from the Nepal Health Research Council Ethical Review Board (reg. no. 101/2018).

Informed Consent Statement: Informed written consent was obtained from the primary caregivers of all children with $\mathrm{CP}$ involved in the study.

Data Availability Statement: The data presented in this study are available on request from the corresponding author. The data are not publicly available due to privacy/ethical restrictions.

Acknowledgments: We would like to express our heartfelt thanks to all caregivers and children for their valuable time and participation in the study activities. We would like to thank Amrit Devkota from District hospital of Gorkha, Nepal, Manik Chandra Das, Tasneem Karim, and Arifuzzaman Khan from CSF Global Bangladesh for their time and effort in the clinical assessment, registration of children in NCPR and data collection process. We would also like to acknowledge the CSF Global team in Nepal for their intensive support in the study implementation and for supporting the children with CP and their families with information and in accessing services along with a strong referral system.

Conflicts of Interest: The authors declare no conflict of interest. The funders had no role in the design of the study; in the collection, analyses, or interpretation of data; in the writing of the manuscript, or in the decision to publish the results.

\section{References}

1. Rosenbaum, P.; Paneth, N.; Leviton, A.; Goldstein, M.; Bax, M.; Damiano, D.; Dan, B.; Jacobsson, B. A report: The definition and classification of cerebral palsy April 2006. Dev. Med. Child Neurol. Suppl. 2007, 109, 8-14.

2. Jahan, I.; Muhit, M.; Karim, T.; Smithers-Sheedy, H.; Novak, I.; Jones, C.; Badawi, N.; Khandaker, G. What makes children with cerebral palsy vulnerable to malnutrition? Findings from the Bangladesh cerebral palsy register (BCPR). Disabil. Rehabil. 2019, 41, 2247-2254. [CrossRef]

3. Jahan, I.; Muhit, M.; Hardianto, D.; Karim, T.; Al Imam, M.H.; Das, M.C.; Smithers-Sheedy, H.; Badawi, N.; Khandaker, G. Nutritional status of children with cerebral palsy in remote Sumba Island of Indonesia: A community-based key informants study. Disabil. Rehabil. 2019, 43, 1819-1828. [CrossRef] [PubMed]

4. Karim, T.; Jahan, I.; Dossetor, R.; Giang, N.T.; Van Anh, N.T.; Dung, T.Q.; Chau, C.M.; Van Bang, N.; Badawi, N.; Khandaker, G.; et al. Nutritional status of children with cerebral palsy-Findings from prospective hospital-based surveillance in Vietnam indicate a need for action. Nutrients 2019, 11, 2132. [CrossRef] [PubMed] 
5. Power, R.; Muhit, M.; Heanoy, E.; Karim, T.; Badawi, N.; Akhter, R.; Khandaker, G. Health-related quality of life and mental health of adolescents with cerebral palsy in rural Bangladesh. PLoS ONE 2019, 14, e0217675. [CrossRef] [PubMed]

6. Jahan, I.; Karim, T.; Das, M.C.; Muhit, M.; Mcintyre, S.; Smithers-Sheedy, H.; Badawi, N.; Khandaker, G. Mortality in children with cerebral palsy in rural Bangladesh: A population-based surveillance study. Dev. Med. Child Neurol. 2019, 61, 1336-1343. [CrossRef]

7. Aggarwal, S.; Chadha, R.; Pathak, R. Nutritional status and growth in children with cerebral palsy: A review. Int. J. Med. Sci. Public Health 2015, 4, 737-744. [CrossRef]

8. Aydin, K.; Akbas, Y.; Unay, B.; Arslan, M.; Cansu, A.; Sahin, S.; Dilber, C.; Gungor, O.; Aksoy, A.; Yuksel, D.; et al. A multicenter cross-sectional study to evaluate the clinical characteristics and nutritional status of children with cerebral palsy. Clin. Nutr. ESPEN 2018, 26, 27-34. [CrossRef] [PubMed]

9. Kakooza-Mwesige, A.; Tumwine, J.K.; Eliasson, A.C.; Namusoke, H.K.; Forssberg, H. Malnutrition is common in Ugandan children with cerebral palsy, particularly those over the age of five and those who had neonatal complications. Acta Paediatr. 2015, 104, 1259-1268. [CrossRef] [PubMed]

10. Léonard, M.; Dain, E.; Pelc, K.; Dan, B.; De Laet, C. Nutritional status of neurologically impaired children: Impact on comorbidity. Arch. Pediatr. 2020, 27, 95-103. [CrossRef]

11. Wang, F.; Cai, Q.; Shi, W.; Jiang, H.; Li, N.; Ma, D.; Wang, Q.; Luo, R.; Mu, D. A cross-sectional survey of growth and nutritional status in children with cerebral palsy in West China. Pediatr. Neurol. 2016, 58, 90-97. [CrossRef]

12. Karagiozoglou-Lampoudi, T.; Daskalou, E.; Vargiami, E.; Zafeiriou, D. Identification of feeding risk factors for impaired nutrition status in paediatric patients with cerebral palsy. Acta Paediatr. 2012, 101, 649-654. [CrossRef]

13. Khandaker, G.; Muhit, M.; Karim, T.; Smithers-Sheedy, H.; Novak, I.; Jones, C.; Badawi, N. Epidemiology of cerebral palsy in Bangladesh: A population-based surveillance study. Dev. Med. Child Neurol. 2019, 61, 601-609. [CrossRef]

14. Oskoui, M.; Coutinho, F.; Dykeman, J.; Jetté, N.; Pringsheim, T. An update on the prevalence of cerebral palsy: A systematic review and meta-analysis. Dev. Med. Child Neurol. 2013, 55, 509-519. [CrossRef]

15. Herrera-Anaya, E.; Angarita-Fonseca, A.; Herrera-Galindo, V.M.; Martínez-Marín, R.D.; Rodríguez-Bayona, C.N. Association between gross motor function and nutritional status in children with cerebral palsy: A cross-sectional study from Colombia. Dev. Med. Child Neurol. 2016, 58, 936-941. [CrossRef]

16. Banskota, B.; Shrestha, S.; Rajbhandari, T.; Banskota, A.; Spiegel, D. A snapshot of 1001 children presenting with cerebral palsy to a children's disability hospital. J. Nepal. Health Res. Counc. 2015, 13, 31-37. [PubMed]

17. Thapa, R. Retrospective descriptive study of cerebral palsy in Nepal. J. Autism Dev. Disord. 2016, 46, 2285-2291. [CrossRef] [PubMed]

18. LeBrun, D.G.; Banskota, B.; Banskota, A.K.; Rajbhandari, T.; Baldwin, K.D.; Spiegel, D.A. Socioeconomic status influences functional severity of untreated cerebral palsy in Nepal: A prospective analysis and systematic review. Clin. Orthop. Relat. Res. 2019, 477, 10. [CrossRef] [PubMed]

19. Local Level Authorities, Nepal. 2017. Available online: http://rapnepal.com/sites/default/files/report-publication/local_govt_ of_75_districts23mar2017.pdf (accessed on 18 July 2021).

20. Central Bureau of Statistics. National Population and Housing Census 2011 (National Report). 2012. Available online: https: // unstats.un.org/unsd/demographic-social/census/documents/Nepal/Nepal-Census-2011-Vol1.pdf (accessed on 18 July 2021).

21. Mackey, S.; Murthy, G.V.; Muhit, M.A.; Islam, J.J.; Foster, A. Validation of the key informant method to identify children with disabilities: Methods and results from a pilot study in Bangladesh. J. Trop. Pediatr. 2012, 58, 269-274. [CrossRef]

22. Cans, C. Surveillance of cerebral palsy in Europe: A collaboration of cerebral palsy surveys and registers. Dev. Med. Child Neurol. 2000, 42, 816-824. [CrossRef]

23. ACPR. Report of the Australian Cerebral Palsy Register, Birth Years 1995-2012. Australia, 12 December 2018. Available online: https:/ / www.ausacpdm.org.au/resources/australian-cerebral-palsy-register/ (accessed on 18 July 2021).

24. Rosenbaum, P.L.; Palisano, R.J.; Bartlett, D.J.; Galuppi, B.E.; Russell, D.J. Development of the gross motor function classification system for cerebral palsy. Dev. Med. Child Neurol. 2008, 50, 249-253. [CrossRef]

25. Eliasson, A.C.; Krumlinde-Sundholm, L.; Rösblad, B.; Beckung, E.; Arner, M.; Öhrvall, A.M.; Rosenbaum, P. The Manual Ability Classification System (MACS) for children with cerebral palsy: Scale development and evidence of validity and reliability. Dev. Med. Child. Neurol. 2006, 48, 549-554. [CrossRef] [PubMed]

26. Pennington, L.; Virella, D.; Mjøen, T.; da Graça Andrada, M.; Murray, J.; Colver, A.; Himmelmann, K.; Rackauskaite, G.; Greitane, A.; Prasauskiene, A.; et al. Development of The Viking Speech Scale to classify the speech of children with cerebral palsy. Res. Dev. Disabil. 2013, 34, 3202-3210. [CrossRef]

27. Hidecker, M.J.; Paneth, N.; Rosenbaum, P.L.; Kent, R.D.; Lillie, J.; Eulenberg, J.B.; Chester, J.R.K.E.; Johnson, B.; Michalsen, L.; Evatt, M.; et al. Developing and validating the Communication Function Classification System for individuals with cerebral palsy. Dev. Med. Child Neurol. 2011, 53, 704-710. [CrossRef]

28. World Health Organization (WHO). WHO Expert Committee on Physical Status: The Use and Interpretation of Anthropometry. Switzerland, 1995. Available online: https://apps.who.int/iris/bitstream/handle/10665/37003/WHO_TRS_854.pdf;jsessionid= 08FC457AB2A52609E61DE1F499FAF4BE?sequence=1 (accessed on 18 July 2021).

29. Stevenson, R.D. Use of segmental measures to estimate stature in children with cerebral palsy. Arch. Pediatr. Adolesc. Med. 1995, 149, 658-662. [CrossRef] 
30. Ministry of Health, Nepal; New ERA; ICF. Nepal Demographic and Health Survey 2016. Ministry of Health, Nepal: Kathmandu, Nepal, 2017. Available online: https:/ /www.dhsprogram.com/pubs/pdf/fr336/fr336.pdf (accessed on 18 July 2021).

31. Polack, S.; Adams, M.; O’banion, D.; Baltussen, M.; Asante, S.; Kerac, M.; Gladstone, M.; Zuurmond, M. Children with cerebral palsy in Ghana: Malnutrition, feeding challenges, and caregiver quality of life. Dev. Med. Child Neurol. 2018, 60, 914-921. [CrossRef]

32. Ruiz Brunner, M.D.; Cieri, M.E.; Rodriguez Marco, M.P.; Schroeder, A.S.; Cuestas, E. Nutritional status of children with cerebral palsy attending rehabilitation centers. Dev. Med. Child Neurol. 2020, 62, 1383-1388. [CrossRef] [PubMed]

33. Dahlseng, M.O.; Finbråten, A.K.; Júlíusson, P.B.; Skranes, J.; Andersen, G.; Vik, T. Feeding problems, growth and nutritional status in children with cerebral palsy. Acta Paediatr. 2012, 101, 92-98. [CrossRef]

34. Benfer, K.A.; Weir, K.A.; Bell, K.L.; Ware, R.S.; Davies, P.S.; Boyd, R.N. Oropharyngeal dysphagia and gross motor skills in children with cerebral palsy. Pediatrics 2013, 131, e1553-e1562. [CrossRef] [PubMed]

35. Sung, K.H.; Chung, C.Y.; Lee, K.M.; Cho, B.C.; Moon, S.J.; Kim, J.; Park, M.S. Differences in body composition according to gross motor function in children with cerebral palsy. Arch. Phys. Med. Rehabil. 2017, 98, 2295-2300. [CrossRef]

36. Walker, J.L.; Bell, K.L.; Boyd, R.N.; Davies, P.S. Energy requirements in preschool-age children with cerebral palsy. Am. J. Clin. Nutr. 2012, 96, 1309-1315. [CrossRef] [PubMed]

37. Scarpato, E.; Staiano, A.; Molteni, M.; Terrone, G.; Mazzocchi, A.; Agostoni, C. Nutritional assessment and intervention in children with cerebral palsy: A practical approach. Int. J. Food Sci. Nutr. 2017, 68, 763-770. [CrossRef] [PubMed]

38. Mlinda, S.J.; Leyna, G.H.; Massawe, A. The effect of a practical nutrition education programme on feeding skills of caregivers of children with cerebral palsy at Muhimbili National Hospital, in Tanzania. Child Care Health Dev. 2018, 44, 452-461. [CrossRef]

39. Donkor, C.M.; Lee, J.; Lelijveld, N.; Adams, M.; Baltussen, M.M.; Nyante, G.G.; Kerac, M.; Polack, S.; Zuurmond, M. Improving nutritional status of children with Cerebral palsy: A qualitative study of caregiver experiences and community-based training in Ghana. Food Sci. Nutr. 2019, 7, 35-43. [CrossRef] [PubMed]

40. Banks, L.M.; Walsham, M.; Neupane, S.; Neupane, S.; Pradhananga, Y.; Maharjan, M.; Blanchet, K.; Kuper, H. Access to social protection among people with disabilities: Mixed methods research from Tanahun, Nepal. Eur. J. Dev. Res. 2019, 31, 929-956. [CrossRef] 